\title{
TWO REMARKS ON THE HOMOLOGY OF GROUP EXTENSIONS
}

\author{
Dedicated to the memory of Hanna Neumann \\ PETER HILTON and URS STAMMBACH
}

(Received 28 July 1972)

\section{Introduction}

In this note we apply a particular technique to obtain information on the homology homomorphism $\varepsilon_{*}: H_{*}(G ; A) \rightarrow H_{*}(Q ; A)$ associated with a group extension

$$
N>\stackrel{\mu}{\longrightarrow} G \stackrel{\varepsilon}{\longrightarrow} \longrightarrow Q
$$

and a $Q$-module $A$. The technique consists of using $\varepsilon$ itself to pull-back $(0.1)$; that is, we construct the pull-back extension induced from (0.1) by $\varepsilon$. This, however, is nothing but the semidirect product, $N\} G$, of $N$ and $G$, with $G$ operating on the left on $N$ by conjugation. Thus we obtain from (0.1) the commutative diagram

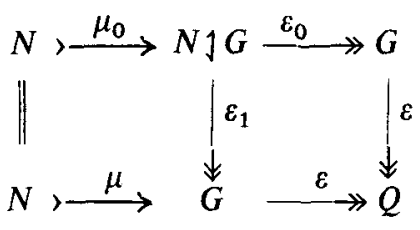

where $\varepsilon_{0}$ is the projection and $\varepsilon_{1}$ is the multiplication $\varepsilon_{1}(n, x)=n x, n \in N, x \in G$. We now apply the Lyndon-Hochschild-Serre spectral sequence functor to $(0.2)$ and carry out computations in dimensions 2 and 3.

In Chapter 1 we are concerned to study the kernel of $\varepsilon_{*}: H_{2}(G ; A) \rightarrow H_{2}(Q ; A)$. Thus we seek to extend the standard 5-term exact sequence

$$
H_{2}(G ; A) \rightarrow H_{2}(Q ; A) \rightarrow N_{a b} \otimes_{Q} A \rightarrow H_{1}(G ; A) \rightarrow H_{1}(Q ; A) \rightarrow 0
$$

one place to the left. We obtain, by the method outlined above, a generalization to arbitrary coefficients of a theorem proved by Nomura [6], by topological methods, for integer coefficients. The rest of Chapter 1 is concerned with refinements 
of the result and with the relation of the result to Ganea's extension of (0.3) in [5] in the case of a central extension (0.1) and integer coefficients.

In Chapter 2 we refer to the 8-term sequence obtained in [4] which extends (0.3) three places to the left, again in the case of a central extension and integer coefficients. The extended sequence (2.1) is then extended a further two places to the left, provided thet we replace $\varepsilon_{*}: H_{3} G \rightarrow H_{3} Q$, which is the initial homomorphism of the 8-term sequence, by an induced homomorphism

$$
\bar{\varepsilon}: H_{3} G / B \rightarrow H_{3} Q,
$$

where $B$ is an explicitly described subgroup of $H_{3} G$. Once again, the technique is as described earlier, but we make decisive use, first, of the fact that the spectral sequence of a direct product collapses (if $N$ is central, then the semi-direct product $N\} G$ is just the direct product $N \times G$ ) and, second, of André's calculation [1] of the differential $d_{2}$ in the Lyndon-Hochschild-Serre spectral sequence.

The rest of Chapter 2 consists of a discussion of the subgroup $B$ of $\mathrm{H}_{3} G$ and the associated quotient group $H_{3} G / B$. We remark that a similar extension of the 8-term sequence (2.1) was obtained in [2] by topological arguments, and that this extension also involved factoring out a certain subgroup $B^{\prime}$ of $H_{3} G$. It is always the case that $B^{\prime} \subseteq B$, but we show by an example that, in general, $B^{\prime} \neq B$. Of course, this difference in the third term of the two extensions of (2.1) (i.e (2.2) of this paper and (1.5) of [2]) is compensated by a complementary difference in their second term.

The fact that an exact sequence of the type of (2.2) must exist was first discovered by Gut.

\section{Nomura's Theorem}

1.1 Let $E: N>\stackrel{\mu}{\longrightarrow} G \stackrel{\varepsilon}{\longrightarrow} \ggg Q$ be an exact sequence of groups. Given any $\tau: P \rightarrow Q$ we form the pull-back

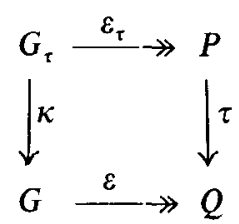

and hence an induced sequence

$$
\tau^{*} E: N>\stackrel{\mu_{\tau}}{\longrightarrow} G_{\tau} \stackrel{\varepsilon_{\tau}}{\longrightarrow} P
$$

together with a map

$$
(1, \kappa, \tau): \tau^{*} E \rightarrow E
$$

It follows from the pull-back property that any map $(\alpha, \beta, \tau): E^{\prime} \rightarrow E$ of sequences, 


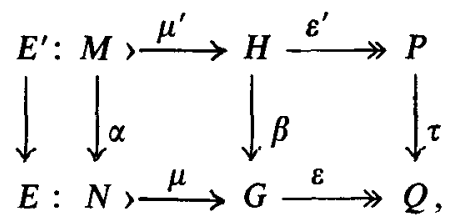

factors uniquely through $\tau^{*} E$ as

$$
(\alpha, \beta, \tau)=(1, \kappa, \tau) \circ\left(\alpha, \beta^{\prime}, 1\right)
$$

for a uniquely determined $\beta^{\prime}: H \rightarrow G_{\boldsymbol{\tau}}$.

Now consider, in particular, $\varepsilon^{*} E$. It is easy to see that $\varepsilon^{*} E$ is just

$$
N>\stackrel{\mu_{0}}{\longrightarrow} N 1 G \stackrel{\varepsilon_{0}}{\longrightarrow} G,
$$

where $N\} G$ is the semi-direct product of $N$ and $G$, with $G$ operating on the left on $N$ by inner automorphism. Moreover, $\mu_{0}$ is the canonical embedding, $\varepsilon_{0}$ the canonical projection; and, further, there is a canonical splitting $\lambda_{0}: G \rightarrow N j G$ of (1.4) such that $\varepsilon_{0} \lambda_{0}=1$, given by $\lambda_{0}(x)=(1, x), x \in G$. We regard the splitting $\lambda_{0}$ as part of the structure of the semi-direct product.

The map $\varepsilon^{*} E \rightarrow E$ is $\left(1, \varepsilon_{1}, \varepsilon\right)$,

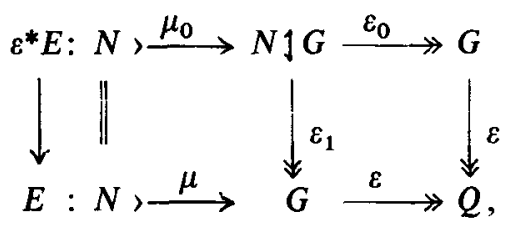

where $\left.\varepsilon_{1}: N\right\} G \rightarrow G$ is the multiplication map, given by $\varepsilon_{1}(n, x)=n x, n \in N, x \in G$.

Now, for any $\sigma: P \rightarrow G, \sigma^{*} \varepsilon^{*} E$ splits. Indeed we obtain

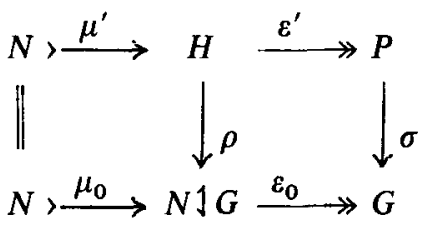

together with a splitting $\lambda: P \rightarrow H$ such that $\rho \lambda=\lambda_{0} \sigma$. We prove a strong converse of this.

Propositron 1.1. The sequence $\varepsilon^{*} E$ is universal for splitting exact sequences over $E$. Precisely, if in (1.3), $E^{\prime}$ splits by $\lambda: P \rightarrow H$ then we have a unique factorization.

$$
(\alpha, \beta, \tau)=\left(1, \varepsilon_{1}, \varepsilon\right) \circ(\alpha, \rho, \sigma),
$$

where $\rho$ satisfies

$$
\rho \hat{\lambda}=\lambda_{0} \sigma
$$


Proof. Set $\sigma=\beta \lambda: P \rightarrow G$. Then $\varepsilon \sigma=\varepsilon \beta \lambda=\tau \varepsilon^{\prime} \lambda=\tau$.

Now $\varepsilon \sigma \varepsilon^{\prime}=\tau \varepsilon^{\prime}=\varepsilon \beta$. Thus, by the pull-back property, there exists a unique $\rho: H \rightarrow N j G$, given by $\varepsilon_{0} \rho=\sigma \varepsilon^{\prime}, \varepsilon_{1} \rho=\beta$. One easily proves that $\rho \mu^{\prime}=\mu_{0}$. Moreover, observing that $\varepsilon_{1} \lambda_{0}=1$, it follows readily that $\rho \lambda=\lambda_{0} \sigma$. Conversely, from (1.7) and (1.8) we infer that $\sigma=\varepsilon_{1} \rho \lambda=\beta \lambda$, and then $\rho$ is determined by the equations $\varepsilon_{0} \rho=\sigma \varepsilon^{\prime}, \varepsilon_{1} \rho=\beta$.

Note. In fact, $\rho$ is given by $\rho x=\left(\beta x\left(\beta \lambda \varepsilon^{\prime} x\right)^{-1}, \beta \lambda \varepsilon^{\prime} x\right), x \in H$.

Corollary 1.2. If $\alpha=1$, then $E^{\prime}=\sigma^{*} \varepsilon^{*} E$.

Finally, we remark that, if $N$ is central in $G$, then (1.4) reduces to the direct product

$$
N>\stackrel{\mu_{0}}{\longrightarrow} N \times G \stackrel{\varepsilon_{0}}{\longrightarrow} G
$$

we will exploit this in Chapter 2.

1.2 In this section we prove the following theorem, generalizing (to arbitrary coefficient modules) a theorem proved by Nomura [6] by topological methods.

THEOREM 1.3. Given the short exact sequence of groups

$$
E: N \stackrel{\mu}{\longrightarrow} G \stackrel{\varepsilon}{\longrightarrow} Q
$$

and the $Q$-module $A$, there exists an exact sequence ker $\varepsilon_{0^{*}} \stackrel{\varepsilon_{1^{\star}}}{\longrightarrow} H_{2}(G ; A) \stackrel{\varepsilon_{*}}{\longrightarrow} H_{2}(Q ; A) \rightarrow N_{a b} \otimes_{Q} A \rightarrow H_{1}(G ; A) \rightarrow H_{1}(Q ; A) \rightarrow 0$, where $\varepsilon_{0}, \varepsilon_{1}$ are as in (1.5) and $\left.\varepsilon_{0^{*}}: H_{2}(N\} G ; A\right) \rightarrow H_{2}(G ; A)$.

Proof. Of course only exactness at $H_{2}(G ; A)$ is in question. We prove this by considering the map of Lyndon-Hochschild-Serre (henceforth, L-HS) spectral sequences induced by (1.5). For $\varepsilon^{*} E$ we have a spectral sequence $\left\{\widetilde{E}_{r}^{p q}\right\}$, such that

$$
\tilde{E}_{2}^{p q}=H_{p}\left(G ; H_{q}(N ; A)\right) ;
$$

and there is a filtration $\widetilde{F}_{0} \subseteq \widetilde{F}_{1} \subseteq \widetilde{F}_{2}$ of $\left.H_{2}(N\} G ; A\right)$ such that

$$
\tilde{F}_{0}=\tilde{E}_{\infty}^{02}, \widetilde{F}_{1} / \tilde{F}_{0}=\tilde{E}_{\infty}^{11}, \widetilde{F}_{2} / \tilde{F}_{1}=\tilde{E}_{\infty}^{20}, \widetilde{F}_{2}=H_{2}(N j G ; A)
$$

Moreover, $\tilde{F}_{1}=\operatorname{ker} \varepsilon_{0^{*}}: H_{2}(N 1 G ; A) \rightarrow H_{2}(G ; A)$.

Similarly, for the extension $E$ we have a spectral sequence $\left\{E_{r}^{p q}\right\}$ such that

$$
E_{2}^{p q}=H_{p}\left(Q ; H_{q}(N ; A)\right) ;
$$

and there is a filtration $F_{0} \subseteq F_{1} \subseteq F_{2}$ of $H_{2}(G ; A)$ such that

$$
F_{0}=E_{\infty}^{02}, F_{1} / F_{0}=E_{\infty}^{11}, F_{2} / F_{1}=E_{\infty}^{20}, F_{2}=H_{2}(G ; A) .
$$

Moreover, $F_{1}=\operatorname{ker} \varepsilon_{*}: H_{2}(G ; A) \rightarrow H_{2}(Q ; A)$.

Thus we must prove that $\left(1, \varepsilon_{1}, \varepsilon\right): \varepsilon^{*} E \rightarrow E$ induces a surjection $\tilde{F}_{1} \rightarrow F_{1}$. We have the diagram, with exact rows, 


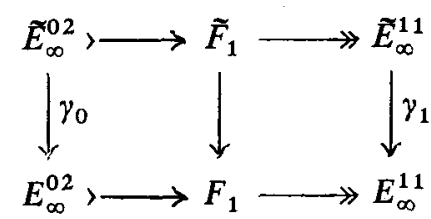

so it suffices to show that $\left(1, \varepsilon_{1}, \varepsilon\right)$ induces surjections

$$
\gamma_{0}: \widetilde{E}_{\infty}^{02} \rightarrow E_{\infty}^{02}, \quad \gamma_{1}: \widetilde{E}_{\infty}^{11} \rightarrow E_{\infty}^{11} .
$$

Now $\tilde{E}_{2}^{02}=H_{2}(N ; A)_{G}, E_{2}^{02}=H_{2}(N ; A)_{Q}$, so that $\left(1, \varepsilon_{1}, \varepsilon\right)$ induces an isomorphism $\tilde{E}_{2}^{02} \rightarrow E_{2}^{02}$. Since $\tilde{E}_{\infty}^{02}, E_{\infty}^{02}$ are quotients of $\widetilde{E}_{2}^{02}, E_{2}^{02}$ respectively, it follows that $\gamma_{0}$ is surjective.

Again

$$
\tilde{E}_{2}^{11}=H_{1}\left(G ; H_{1}(N ; A)\right), E_{2}^{11}=H_{1}\left(Q ; H_{1}(N ; A)\right),
$$

so that $\left(1, \varepsilon_{1}, \varepsilon\right)$ induces a surjection $\widetilde{E}_{2}^{11} \rightarrow E_{2}^{11}$. Since $\tilde{E}_{\infty}^{11}, E_{\infty}^{11}$ are quotients of $\tilde{E}_{2}^{11}, E_{2}^{11}$ respectively, it follows that $\gamma_{1}$ is surjective. Thus the theorem is proved.

Notes. (a) It may be observed from the proof that we may replace the first term of the exact sequence by

$$
\operatorname{ker} \varepsilon_{\tau}^{*} \stackrel{\kappa_{*}}{\longrightarrow} H_{2}(G ; A),
$$

for any surjective $\tau: P \rightarrow Q$, where (see (1.1), (1.2))

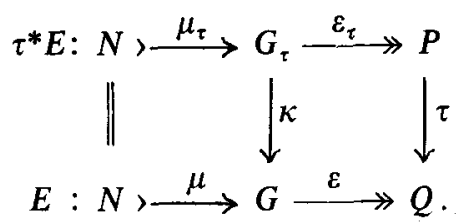

(b) Since (1.4) splits it follows that, in the spectral sequence $\left\{\tilde{E}_{r}^{p t}\right\}$, the differential $\tilde{d}_{r} ; \widetilde{E}_{r}^{p 0} \rightarrow \widetilde{E}_{r}^{p-r, r-1}$ is always zero. Thus $\widetilde{E}_{2}^{11}=\widetilde{E}_{\infty}^{11}$ and $\widetilde{E}_{3}^{02}=\widetilde{E}_{\infty}^{02}$. We therefore obtain from the top row of (1.10) the exact sequence

$$
H_{2}\left(G ; H_{1}(N ; A)\right) \stackrel{\tilde{d}_{2}}{\longrightarrow} H_{2}(N ; A)_{G} \rightarrow \operatorname{ker} \varepsilon_{0^{*}} \rightarrow H_{1}\left(G ; H_{1}(N ; A)\right) \rightarrow 0 .
$$

1.3 Nomura states in the introduction to [6] that the exact sequence of Theorem 1.3, with $A=\mathbb{Z}$, provides a generalization of Ganea's result [5] for central extensions. He does, in fact, reprove Ganea's result in [6], but Theorem 1.3 does not immediately yield that result. For if we suppose that $N$ is central in $G$, then, as pointed out in 1.1, N1G becomes the direct product $N \times G$ and ker $\varepsilon_{0^{*}}$ * admits a natural direct sum decomposition

$$
\text { ker } \varepsilon_{0 *}=H_{2} N \oplus\left(N \otimes G_{a b}\right) \text {. }
$$


Moreover $\varepsilon_{1 *} \mid H_{2} N$ is just $\mu_{*}$ and $\varepsilon_{1 *} \mid\left(N \otimes G_{a b}\right)$ is the Ganea term $\chi$ [3], which Ganea proved, by topological arguments, could be added to the left of the 5-term homology sequence, with integer coefficients. Explicitly, Theorem 1.3 yields, for central extensions, the exactness of

$$
H_{2} N \oplus\left(N \otimes G_{a b}\right) \stackrel{\varepsilon_{1 *}=\left\langle\mu_{*}, \chi\right\rangle}{\rightarrow} H_{2} G \rightarrow H_{2} Q \rightarrow \cdots,
$$

while Ganea proved the exactness of

$$
\mathrm{N} \otimes G_{a b} \stackrel{\chi}{\longrightarrow} \mathrm{H}_{2} \mathrm{G} \rightarrow \mathrm{H}_{2} Q \rightarrow \cdots .
$$

Thus, to deduce (1.14) from (1.13) one must prove

$$
\mu_{*} H_{2} N \subseteq \chi\left(N \otimes G_{a b}\right) .
$$

Now naturality yields a commutative diagram

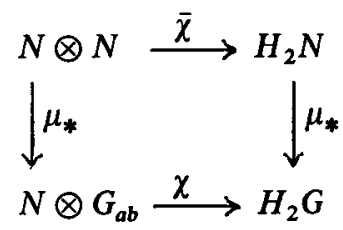

where $\bar{\chi}$ is the Ganea term for the central extension $N \longrightarrow N \longrightarrow 1$. Thus (1.15) follows immediately from

Proposition 14. $\bar{\chi}: N \otimes N \rightarrow H_{2} N$ is surjective.

NOTE. This proposition follows immediately if one assumes Ganea's result. However, to deduce Ganea's result from Theorem 1.3 we should provide an independent proof of the proposition.

Proof. It is sufficient to consider the case where $N$ is finitely generated, since we may then complete the proof by a direct limit argument. Since the case of $N$ cyclic is trivial, the proof is completed by observing that, if $N=N_{1} \oplus N_{2}$, then $\bar{\chi} \mid N_{1} \otimes N_{2}$ maps $N_{1} \otimes N_{2}$ identically onto $N_{1} \otimes N_{2} \subseteq H_{2} N$.

1.4. We return to the general case. In the light of (1.11) which may be regarded as a generalization of (1.12), it is reasonable to ask when we may replace the term ker $\varepsilon_{0 *} \stackrel{\varepsilon_{1 *}}{\longrightarrow} H_{2} G$ in Theorem 2.3 by $H_{1}\left(G ; N_{a b}\right) \stackrel{\chi}{\longrightarrow} H_{2} G$, for some suitably defined $\chi$. We give below a sufficient condition which, of course, includes the case where $N$ is central in $G$. However, we first draw a trivial inference from Theorem 1.3 and (1.11).

Proposition 1.5. If $\tilde{d}_{2}: H_{2}\left(G ; H_{1}(N ; A)\right) \rightarrow H_{2}(N ; A)_{G}$ is surjective, (e.g., if $\left.H_{2}(N, A)_{G}=0\right)$, we have an exact sequence

$$
H_{1}\left(G ; H_{1}(N ; A)\right) \rightarrow H_{2}(G ; A) \rightarrow H_{2}(Q ; A) \rightarrow \cdots .
$$


To describe the sufficient condition referred to above, we present the extension $N \gg \longrightarrow G \longrightarrow Q$ (see (2.5) of [3]). Thus if $R \stackrel{\iota}{\longrightarrow} F \stackrel{\pi}{\longrightarrow} G$ is a free presentation of $G$ we set $S=\pi^{-1} N$, and

$$
\begin{aligned}
& R \longmapsto S \longrightarrow N, \\
& S \gg S \longrightarrow Q
\end{aligned}
$$

are free presentations of $N, Q$ respectively.

The condition we impose is

$$
[[F, S], S] \subseteq[F, R] .
$$

We first prove

LEMMA 1.6. The condition (1.17) is independent of the choice of presentation.

Proof. If also $R^{\prime}>\stackrel{\iota^{\prime}}{\longrightarrow} F^{\prime} \stackrel{\pi^{\prime}}{\longrightarrow} G$ presents $G$, and $S^{\prime}=\pi^{\prime-1}(N)$, there is a homomorphism $\phi: F \rightarrow F^{\prime}$ such that

$$
\pi^{\prime} \phi=\pi
$$

Plainly $\phi S \subseteq S^{\prime}, \phi R \subseteq R^{\prime}$. Moreover, $\phi$ is determined modulo a function $F \rightarrow R^{\prime}$. It follows that the homomorphism

$$
\psi_{0}:[F, F] \rightarrow\left[F^{\prime}, F^{\prime}\right] /\left[F^{\prime}, R^{\prime}\right]
$$

induced by $\phi$ is uniquely determined. We conclude, by standard arguments of a homological-algebraic type, that, in fact, $\phi$ induces an isomorphism

$$
\psi:[F, F] /[F, R] \stackrel{\sim}{\rightarrow}\left[F^{\prime}, F^{\prime}\right] /\left[F^{\prime}, R^{\prime}\right] .
$$

Now $\phi[[F, S], S] \subseteq\left[\left[F^{\prime}, S^{\prime}\right], S^{\prime}\right]$. Suppose that $\left[\left[F^{\prime}, S^{\prime}\right], S^{\prime}\right] \subseteq\left[F^{\prime}, R^{\prime}\right]$ and let $x \in[[F, S], S]$. Then $\psi(x[F, R])$ is the neutral element of $\left[F^{\prime}, F^{\prime}\right] /\left[F^{\prime}, R^{\prime}\right]$ so that $x \in[F, R]$. Thus $[[F, S], S] \subseteq[F, R]$ and the lemma is proved.

We remark that the condition $[[F, S], S] \subseteq[F, R]$ is certainly satisfied by a central extension. However it is also plain that it is satisfied when $N$ is commutative and $\mathrm{H}_{2} \mathrm{~N}=0$ (or $\mathrm{H}_{2} \mathrm{G}=0$ ), so that it is more general than centrality.

We prove

THEOREM 1.7. Let $N>\stackrel{\mu}{\longrightarrow} \dot{G} \stackrel{\varepsilon}{\longrightarrow} Q$ be an extension satisfying (1.17), with $N$ commutative. Then there is a natural exact sequence

$$
H_{1}(G ; N) \stackrel{\chi}{\longrightarrow} H_{2} G \stackrel{\varepsilon_{*}}{\longrightarrow} H_{2} Q
$$

Proof. Since ker $\varepsilon_{*}=(R \cap[F, S]) /[F, R]$, it is sufficient to exhibit a natural surjection $\chi_{0}: H_{1}(G ; N) \longrightarrow(R \cap[F, S]) /[F, R]$. Consider the diagram 


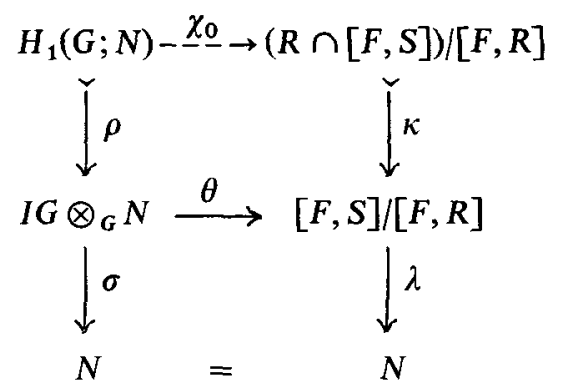

Here the columns are exact and $[F, S] /[F, R]$ is commutative by (1.17). Further $\theta$ is defined by

(1.18) $\theta\left((x R-1) \otimes_{G} y R\right)=[x, y] \bmod [F, R], x \in F, y \in S$.

Now $\left[x, y_{1} y_{2}\right]=\left[x, y_{1}\right]\left[x, y_{2}\right]^{y_{1}} \equiv\left[x, y_{1}\right]\left[x, y_{2}\right] \bmod [F, R]$, by (1.17). Also

$$
[x z, y][z, y]^{-1}=\left[x, z y z^{-1}\right] \text {, }
$$

which shows that $\theta$ respects the defining relations involved in passing to the tensor product over $G$, and so is well-defined by (1.18). It is obvious that $\theta$ is surjective. It is also clear that $\lambda \theta=\sigma$, since $\sigma\left((x R-1) \otimes_{G} y R\right)=[x, y] \bmod R$. Thus $\theta$ induces $\chi_{0}: H_{1}(G ; N) \rightarrow(R \cap[F, S]) /[F, R]$ and $\chi_{0}$ is surjective because $\theta$ is surjective.

That $\chi$ is canonical is proved as follows. If we define $\theta^{\prime}$ as in (1.18), but with respect to the presentation $R^{\prime} \longrightarrow \longrightarrow F^{\prime} \longrightarrow G$, then plainly $\theta^{\prime}=\psi \theta$, where $\psi$ is the isomorphism of the proof of Lemma 1.6. Thus $\chi_{0}^{\prime}=\psi \chi_{0}$. However we use $\psi(R \cap[F, S]) /[F, R]$ to indentify the two kernels, $(R \cap[F, S]) /[F, R]$ and $\left(R^{\prime} \cap\left[F^{\prime}, S^{\prime}\right]\right) /\left[F^{\prime}, R^{\prime}\right]$, of $\varepsilon_{*}: H_{2} G \rightarrow H_{2} Q$. Thus $\chi$ is canonical. A similar type of argument shows $\chi$ to be natural.

We remark that the definition of $\theta$ makes it plain that the homomorphism $\chi$ of this theorem generalizes the Ganea map.

Note. It is easy to deduce from the L-HS spectral sequence that, if $H_{2}(N ; A)_{Q}=0$, then there is an exact sequence

$$
H_{3}(G, A) \rightarrow H_{3}(Q ; A) \rightarrow H_{1}\left(Q ; H_{1}(N ; A)\right) \rightarrow H_{2}(G ; A) \rightarrow H_{2}(Q ; A) \rightarrow \cdots .
$$

The exact sequence of Proposition 1.5 then arises by composing the third homomorphism above with the surjection

$$
H_{1}\left(G ; H_{1}(N ; A)\right) \rightarrow H_{1}\left(Q ; H_{1}(N ; A)\right) .
$$

\section{The 10-term sequence}

2.1 In the second chapter of our note we confine ourselves to the study of central extensions. We recall from [4] that given the central extension 


$$
N>\stackrel{\mu}{\longrightarrow} G \stackrel{\varepsilon}{\longrightarrow} \gg Q
$$

there is an exact sequence

$$
H_{3} G \rightarrow H_{3} Q \rightarrow N \otimes G_{a b} / U \rightarrow H_{2} G \rightarrow H_{2} Q \rightarrow N \rightarrow G_{a b} \rightarrow Q_{a b} \rightarrow 0
$$

Here (see (1.16)) the subgroup $U$ of $N \otimes G_{a b}$ is defined as $\mu_{*}(\operatorname{ker} \bar{\chi})$ where $\bar{\chi}: N \otimes N \rightarrow H_{2} N$ is the Ganea-Pontryagin map and $\mu_{*}: N \otimes N \rightarrow N \otimes G_{a b}$ is induced by $\mu: N \rightarrow G$.

Here we will discuss the continuation to the left of the exact sequence (2.2). We note that such a discussion is already contained in [2]. However, whereas [2] uses topological methods, we proceed in a purely algebraic way. Our main result differs slightly from the main result of [2]; the precise deviation is discussed in 2.5 .

Thus, our main result is as follows.

THEOREM 2.1. Given the central extension $N \longrightarrow G \longrightarrow Q$, there is a natural exact sequence

$$
H_{4} Q \rightarrow A \rightarrow H_{3} G / B \stackrel{\bar{\varepsilon}}{\rightarrow} H_{3} Q \rightarrow C \rightarrow H_{2} G \rightarrow H_{2} Q \rightarrow N \rightarrow G_{a b} \rightarrow Q_{a b} \rightarrow 0
$$

The groups $A, C$ appearing in (2.2) are defined as follows. We define $\mu_{0}: \operatorname{ker} \bar{\chi} \rightarrow N \otimes G_{a b}$ to be the restriction of $\mu_{*}: N \otimes N \rightarrow N \otimes G_{a b}$ to the kernel of $\bar{\chi}: N \otimes N \rightarrow H_{2} N$. Then

$$
A=\operatorname{ker} \mu_{0}, C=\operatorname{coker} \mu_{0} .
$$

Thus, explicitly, $A=\operatorname{ker} \bar{\chi} \cap \operatorname{ker} \mu_{*}, C=\left(N \otimes G_{a b}\right) / \mu_{*}(\operatorname{ker} \bar{\chi})$.

The subgroup $B$ of $H_{3} G$ is defined as follows. Denote by $\varepsilon_{0}: N \times G \rightarrow G$ the projection and by $\varepsilon_{1}: N \times G \rightarrow G$ the multiplication, as in (1.5). Then $B$ is the image under $\varepsilon_{1 *}: H_{3}(N \times G) \rightarrow H_{3} G$ of the group ker $\varepsilon_{0 *}: H_{3}(N \times G) \rightarrow H_{3} G$. Since $\varepsilon \varepsilon_{0}=\varepsilon \varepsilon_{1}$, it is obvious that $\varepsilon_{*}: H_{3} G \rightarrow H_{3} Q$ annihilates $B$ and thus induces $\bar{\varepsilon}: H_{3} G / B \rightarrow H_{3} Q$ in (2.2).

Proof of Theorem 2.1. We have only to define the first two homomorphisms of the sequence (2.2) and prove exactness at $A$ and $H_{3} G / B$. We will again exploit the map of L-HS spectral sequences induced by

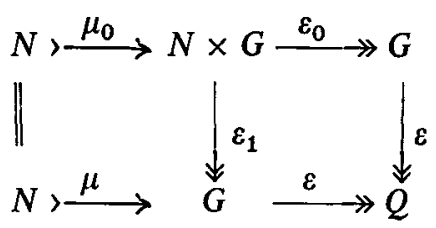

Here we concentrate on dimension 3. Then there is a filtration

such that

$$
F_{0} \subseteq F_{1} \subseteq F_{2} \subseteq F_{3}=H_{3} G
$$

$$
F_{0}=E_{\infty}^{03}, F_{1} / F_{0}=E_{\infty}^{12}, F_{2} / F_{1}=E_{\infty}^{21}, F_{2}=\operatorname{ker} \varepsilon_{*}: H_{3} G \rightarrow H_{3} Q
$$


We note in particular that

$$
\operatorname{ker} \bar{\varepsilon}_{*}=F_{2} / B \text {. }
$$

Now, in the L-HS spectral sequence $\left\{\widetilde{E}_{r}^{p q}\right\}$ associated with

$$
N \gg N \times G \longrightarrow G
$$

all differentials $\tilde{d}_{r}, r \geqq 2$, are trivial,

$$
\widetilde{E}_{2}=\tilde{E}_{\infty} \text {. }
$$

There are also relations analogous to (2.4).

We will study the homomorphisms $\delta_{i}: \widetilde{F}_{i} \rightarrow F_{i}$ induced by (2.3), $i=0,1,2$,

$$
\begin{aligned}
& \tilde{F}_{0} \subseteq \tilde{F}_{1} \subseteq \tilde{F}_{2} \\
& \downarrow \delta_{0}\left|\delta_{1}\right| \delta_{2} \\
& F_{0} \subseteq F_{1} \subseteq F_{2}
\end{aligned}
$$

Naturally, in this study, we utilize the spectral sequence maps $\gamma_{i}: \tilde{E}_{\infty}^{i, 3-i} \rightarrow E_{\infty}^{i, 3-i}$, $i=0,1,2$, so that

$$
\gamma_{0}=\delta_{0}
$$

and there is a commutative diagram, with exact rows

$i=1,2$.

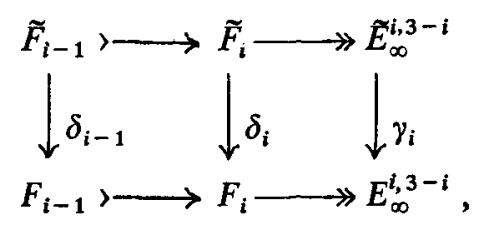

Now $\widetilde{E}_{2}^{03}=H_{3} N, E_{2}^{03}=H_{3} N$. Thus $\gamma_{0}\left(=\delta_{0}\right)$ is clearly surjective. Next, we observe that $\tilde{E}_{2}^{12} \rightarrow E_{2}^{12}$ is just $H_{1}\left(G ; H_{2} N\right) \rightarrow H_{1}\left(Q ; H_{2} N\right)$ and thus certainly surjective. It follows that $\gamma_{1}$ is surjective, so that, by (2.9), $\delta_{1}$ is also surjective.

We now consider (2.9) with $i=2$. Since $\delta_{1}$ is surjective, we know that

$$
\text { coker } \delta_{2} \cong \text { coker } \gamma_{2} \text {. }
$$

However, $B=\operatorname{im} \delta_{2}$, so that, by (2.5) and (2.10),

$$
\text { ker } \bar{\varepsilon}_{*} \cong \operatorname{coker} \gamma_{2} \text {. }
$$

It remains to compute coker $\gamma_{2}$. We know that

$$
\tilde{E}_{\infty}^{2,1}=\tilde{E}_{2}^{2,1}=H_{2}(G ; N) \text {. }
$$

Also $E_{\infty}^{2,1}=E_{3}^{2,1}$. Thus we must study 


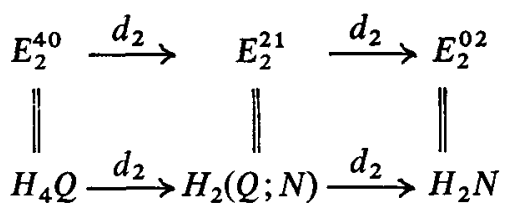

We now require to exploit the computation by André [1] of the differential $d_{2}$ in the L-HS spectral sequence. This computation, applied to the special case of a central extension and trivial coefficients, shows that

$$
d_{2}: \mathrm{H}_{2}(Q ; N) \rightarrow \mathrm{H}_{2} \mathrm{~N}
$$

is the composite of the homomorphism $\beta$, appearing in the 5 -term exact sequence with coefficient module $N$,

$$
H_{2}(G ; N) \stackrel{\varepsilon_{*}}{\longrightarrow} H_{2}(Q ; N) \stackrel{\beta}{\longrightarrow} N \otimes N \stackrel{\mu_{*}}{\longrightarrow} N \otimes G_{a b} \rightarrow N \otimes Q_{a b} \rightarrow 0,
$$

and the Ganea-Pontryagin map

thus,

$$
\bar{\chi}: N \otimes N \rightarrow H_{2} N
$$

$$
d_{2}=\bar{\chi} \beta: H_{2}(Q ; N) \rightarrow H_{2} N
$$

If we regard $\varepsilon_{*}$ in (2.13) as a map $\varepsilon_{* *}: H_{2}(G ; N) \rightarrow \operatorname{ker} d_{2}=\beta^{-1}(\operatorname{ker} \bar{\chi})$, it is then plain that

$$
\text { coker } \varepsilon_{* *}=\beta^{-1}(\operatorname{ker} \bar{\chi}) / \operatorname{ker} \beta \cong \operatorname{ker} \bar{\chi} \cap \operatorname{ker} \mu_{*}=A .
$$

The proof of the theorem is then completed by appeal to (2.11) and the diagram

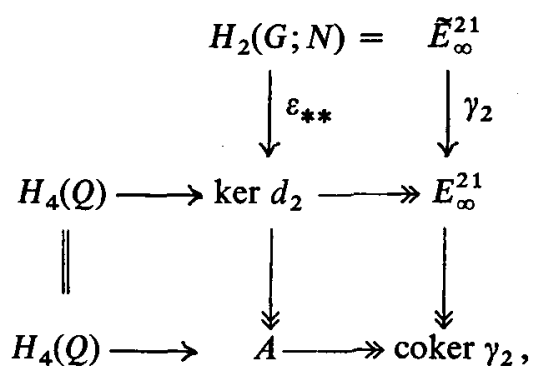

which shows the bottom row to be exact.

2.2 In this section and the next it is convenient to write $G \times N$ instead of $N \times G$. Our concern is with the quotient group $H_{3} G / B$ appearing in (2.2). By the Künneth Theorem one knows that $H_{3}(G \times N)$ fits into the natural short exact sequence

(2.17) $H_{3} N \oplus\left(H_{1} G \otimes H_{2} N\right) \oplus\left(H_{2} G \otimes N\right) \oplus H_{3} G>\rightarrow H_{3}(G \times N) \rightarrow \operatorname{Tor}\left(H_{1} G, N\right)$. 
This sequence splits, but non-naturally in general. It follows that ker $\varepsilon_{0 *}$ fits into the exact sequence

$$
H_{3} N \oplus\left(H_{1} G \otimes H_{2} N\right) \oplus\left(H_{2} G \otimes N\right)>\rightarrow \operatorname{ker} \varepsilon_{0 *} \rightarrow \operatorname{Tor}\left(H_{1} G, N\right),
$$

which also splits non-naturally.

We recall that the subgroup $B \subseteq H_{3} G$ is the image under $\varepsilon_{1^{\star}}: H_{3}(G \times N)$ $\rightarrow H_{3} G$ of ker $\varepsilon_{0 *}$. Here $\varepsilon_{1}: G \times N \rightarrow G$ is the multiplication and $\varepsilon_{0}: G \times N \rightarrow G$ is the projection. It is the purpose of this section to show that

$$
\varepsilon_{1 *}\left(H_{1} G \otimes H_{2} N\right) \subseteq \varepsilon_{1 *}\left(H_{2} G \otimes N\right)
$$

As a consequence we may write

$$
H_{3} G / B=H_{3} G / \varepsilon_{1 *}\left(H_{3} N \oplus H_{2}(G ; N)\right),
$$

in the sense that one first factors out $\varepsilon_{1 *}\left(H_{3} N \oplus\left(H_{2} G \otimes N\right)\right)$, and then factors $\operatorname{Tor}\left(H_{1} G, N\right)$ out of the quotient.

For the proof of (2.19) we consider the diagram

$$
\begin{aligned}
& G \times N \times N \stackrel{1 \times m}{\longrightarrow} G \times N \\
& \varepsilon_{1} \times 1 \downarrow \downarrow \varepsilon_{1} \\
& G \times N \stackrel{\varepsilon_{1}}{\longrightarrow} G \quad G \quad\left(m=\varepsilon_{1} \mid N \times N\right)
\end{aligned}
$$

which is obviously commutative, and the induced square

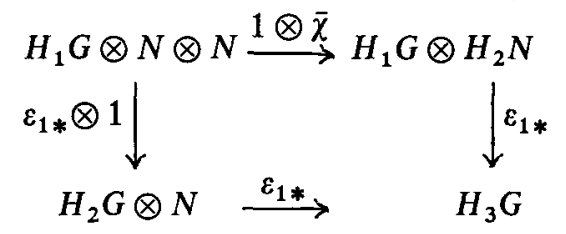

Since $\bar{\chi}: N \otimes N \rightarrow H_{2} N$ is surjective, so is $1 \otimes \bar{\chi}$ and (2.19) now follows immediately.

2.3 We show by a counterexample that in equation (2.20) the term $\mathrm{H}_{3} \mathrm{~N}$ cannot be dropped.

Consider the diagram

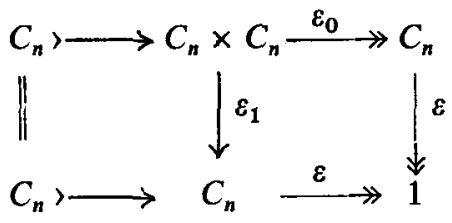

where $C_{n}$ denotes a cyclic group of order $n$. Of course we have $\varepsilon_{1 *}\left(H_{3} C_{n}\right)=H_{3} C_{n}$. However we claim that, if $n$ is even, 


$$
\varepsilon_{1 *}\left(H_{2}\left(C_{n} ; H_{1} C_{n}\right)\right) \neq H_{3} C_{n} .
$$

We first show that $\varepsilon_{1 *} \mid H_{2}\left(C_{n} ; H_{1} C_{n}\right)$ is well-defined. Since $H_{2} C_{n}=0$, we have the exact sequence (see (2.17))

$$
H_{3} C_{n} \oplus H_{3} C_{n}>\rightarrow H_{3}\left(C_{n} \times C_{n}\right) \rightarrow \operatorname{Tor}\left(H_{1} C_{n}, H_{1} C_{n}\right)
$$

Now the embeddings $H_{3} C_{n}>\rightarrow H_{3}\left(C_{n} \times C_{n}\right)$ have natural left inverses induced by the projections $C_{n} \times C_{n} \rightarrow C_{n}$. Hence the splitting of (2.24) is canonical.

Next we use [2; Theorem 2.2] to show that under $\varepsilon_{1 *}$ the subgroup $\operatorname{Tor}\left(H_{1} C_{n}, H_{1} C_{n}\right)=\mathbb{Z}_{n}$ is mapped onto $2 \cdot H_{3} C_{n}=2 \cdot \mathbb{Z}_{n}$. Thus, if $n$ is even,

$$
\varepsilon_{1 *}\left(H_{2}\left(C_{n}: H_{1} C_{n}\right)\right)=2 \cdot H_{3} C_{n} \neq H_{3} C_{n} \text {. }
$$

Finally, comparing sequence (2.2) with sequence (1.5) of [2], we see that in sequence (2.2) the group

$$
H_{3} G / \varepsilon_{1 *}\left(H_{3} N \oplus H_{2}(G ; N)\right)
$$

appears, whereas in sequence (1.5) of [2] we have the group

$$
H_{3} G / \varepsilon_{1 *}\left(H_{2}(G ; N)\right) \text {. }
$$

The above example (2.23) shows that these two groups do not in general agree, so that the two sequences are not in general the same. However, it is not at all difficult to deduce (2.2) from (1.5) of [2].

\title{
References
}

[1] M. André, 'Le $d_{2}$ de la suite spectrale en cohomologie des groupes', C. R. Acad. Sci. Paris 260 (1965), 2669-2671.

[2] B. Eckmann and P. J. Hilton, 'On central group extensions and homology', Comment. Math. Helv. 46 (1971), 345-355.

[3] B. Eckmann, P. J. Hilton and U. Stammbach, 'On the homology theory of central group, extensions; I - The commutator map and stem extensions', Comment. Math. Helv. 47 (1972), 102-122.

[4] B. Eckmann, P. J. Hilton and U. Stammbach, 'On the homology theory of central group extensions; II - The extended exact sequence in the general case', Comment. Math. Helv. 47 (1972), 171-178.

[5] T. Ganea, 'Homologie et extensions centrales de groupes', C. R. Acad. Sci. Paris. 266 (1968) 556-558.

[6] Y. Nomura, 'The Whitney Join and its dual, Osaka J. Math. 7 (1970), 353-373.

\author{
Battelle Seattle Research Center \\ University of Washington, Seattle \\ ETH, Zurich, Switzerland
}

